\title{
PETTIS DECOMPOSITION FOR UNIVERSALLY SCALARLY MEASURABLE FUNCTIONS
}

\author{
ELIZABETH M. BATOR
}

(Communicated by William J. Davis)

\begin{abstract}
It is shown that if $K$ is a compact Hausdorff space, $X$ is a Banach space, and $f: K \rightarrow X^{*}$ is bounded and universally scalarly measurable, then $f$ is $\mu$-Pettis decomposable for every Radon measure $\mu$ on $K$.
\end{abstract}

1. Introduction. A decomposition of scalarly measurable functions was introduced in [2]. If $X$ is a Banach space and $(\Omega, \Sigma, \mu)$ is a finite measure space, a function $h: \Omega \rightarrow X^{*}$ is defined to be $\mu$-weak $k^{*}$ scalarly null if $x h=0$ a.e. $\mu$ for every $x \in X$. We then define a function $f: \Omega \rightarrow X^{*}$ to be $\mu$-Pettis decomposable if there exist functions $g$ and $h$ such that $g$ is $\mu$ Pettis integrable and $h$ is $\mu$-weak* scalarly null and $f=g+h$. Some equivalent conditions for a function to be $\mu$-Pettis decomposable are discussed in [2].

One reason for studying this decomposition is to extend results concerning the Pettis integral that are true for functions taking their ranges in duals of separable Banach spaces, but not true if we remove the separability hypothesis. Note that Pettis integrable functions coincide with Pettis decomposable functions if the range is the dual of a separable Banach space.

It is shown in [8], for example, that if $X$ is a separable Banach space, $K$ a compact Hausdorff space and $f: K \rightarrow X^{*}$ a bounded and universally scalarly measurable function, then $f$ is universally Pettis integrable. The separability hypothesis cannot be removed due to an example of $\mathrm{R}$. Phillips [6] of a function $f:[0,1] \rightarrow l^{\infty}[0,1]$ such that $f$ is scalarly Borel measurable but not Pettis integrable with respect to Lebesgue measure. The intent of this paper is to prove a version of the abovementioned theorem that includes functions taking their ranges in duals of nonseparable Banach spaces by only insisting that the function $f$ be $\mu$-Pettis decomposable for every Radon measure $\mu$ on $K$. This will answer affirmatively a question originally raised in [2].

Let us introduce some definitions and preliminary facts. Let $(\Omega, \Sigma, \mu)$ be a finite measure space and $X$ be a Banach space. A function $f: \Omega \rightarrow X^{*}$ is scalarly measurable if $x^{* *} f$ is measurable for every $x^{* *} \in X^{* *}$, and $f$ is weak scalarly measurable if $x f$ is measurable for every $x \in X$. If $f$ is weak* scalarly measurable, then the evaluation operator $T_{f}: X \rightarrow L^{1}(\mu)$ is given by $T_{f}(x)=x f$ for every

Received by the editors May 29, 1987 and, in revised form, November 23, 1987. Presented January 9, 1988 at the annual meeting of the AMS in Atlanta, Georgia.

1980 Mathematics Subject Classification (1985 Revision). Primary 28B05, 46G10.

Key words and phrases. Banach space, Pettis integral, universally scalarly measurable, Bourgain property.

This research was partially supported by a Faculty Research Grant from North Texas State University. 


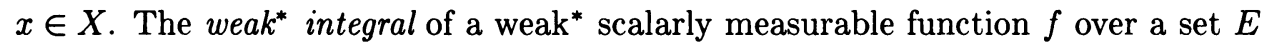
is an element $x_{E}^{*} \in X^{*}$ such that

$$
x_{E}^{*}(x)=\int_{E} x f d u \quad \text { for every } x \in X .
$$

In this case $x_{E}^{*}=w^{*}-\int_{E} f d \mu$.

If $f: \Omega \rightarrow X^{*}$ is bounded and weak ${ }^{*}$ scalarly measurable and if $\Sigma_{0}$ is a sub- $\sigma$ algebra of $\Sigma$, then $f_{0}$ is a weak $k^{*}$ conditional expectation of $f$ with respect to $\Sigma_{0}$, and denoted by $f_{0}=$ weak $^{*}-E\left(f \mid \Sigma_{0}\right)$, if $x f_{0}$ is $\Sigma_{0}$ measurable for every $x \in X$, and for every $A \in \Sigma_{0}$,

$$
\int_{A} x f d u=\int_{A} x f_{0} d u \quad \text { for every } x \in X .
$$

If the function $f$ is scalarly measurable then $f$ is $\mu$-Pettis integrable if for every $E \in \Sigma$, there exists $x_{E}^{*} \in X^{*}$ such that

$$
x^{* *}\left(x_{E}^{*}\right)=\int_{E} x^{* *} f d \mu \quad \text { for every } x^{* *} \in X^{* *} .
$$

In this case, $x_{E}^{*}=P-\int_{E} f d \mu$.

If $K$ is a compact Hausdorff space, then $f: K \rightarrow X^{*}$ is universally scalarly measurable if $f$ is scalarly measurable with respect to all Radon measures on $K$.

Suppose that $Y$ is a subspace of $X$ and $f: \Omega \rightarrow X^{*}$. We then define the function $f_{Y}: \Omega \rightarrow Y^{*}$ by $f_{Y}=T \circ f$, where $T$ is the natural operator from $X^{*}$ to $Y^{*}$.

A subset $K$ of a Banach space $X$ is called weakly precompact if every bounded sequence in $K$ has a weakly Cauchy subsequence. Rosenthal's theorem [9] states that a bounded subset $K$ of a Banach space $X$ is weakly precompact if and only if $K$ does not contain an isomorphic copy of the basis of $l^{1}$.

A subset $\Psi$ of $L^{\infty}(\mu)$ is said to be almost weakly precompact if for every $\varepsilon>0$, there exists a set $A \in \Sigma$ such that $\mu(\Omega \backslash A)<\varepsilon$ and $\left\{f \chi_{A}: f \in \Psi\right\}$ is weakly precompact in $L^{\infty}(\mu)$. In [8], Riddle, Saab, and Uhl show that if $X$ is a separable Banach space, $(\Omega, \Sigma, \mu)$ a finite measure space, and $f: \Omega \rightarrow X^{*}$ a bounded and weak* $^{*}$ scalarly measurable function such that $\left\{x f: x \in B_{X}\right\}$ is almost weakly precompact in $L^{\infty}(\mu)$, then $f$ is $\mu$-Pettis integrable.

A bounded weak* scalarly measurable function $f: \Omega \rightarrow X^{*}$ is said to have the $R S$ property if $\left\{x f: x \in B_{X}\right\}$ is almost weakly precompact in $L^{\infty}(\mu)$. It is shown in Theorem 3 of [2] that if $f$ has the RS property, then $f$ is $\mu$-Pettis decomposable. In [10], Talagrand raises the following question: If $K$ is a compact Hausdorff space and $f: K \rightarrow X^{*}$ is universally scalarly measurable, must $f$ have the RS property for every Radon measure $\mu$ on $K$ ? If the answer to this is yes, then it would immediately follow from Theorem 3 of [2] that every such $f$ would indeed be $\mu$ Pettis decomposable for every Radon measure $\mu$. However, the answer remains unknown. The following proposition does give a partial answer to Talagrand's question.

PROPOSITION 1. Let $K$ be a compact Hausdorff space, $X$ a Banach space, and $f: K \rightarrow X^{*}$ be a bounded, universally scalarly measurable function. If $Y$ is a separable subspace of $X$, then $f_{Y}$ has the $R S$ property. Consequently $\left\{y f: y \in B_{Y}\right\}$ is almost weakly precompact in $L^{\infty}(\mu)$ for every Radon measure $\mu$. 
ProOF. Let $Y$ be a separable subspace of $X$. Since $f$ is universally scalarly measurable, it is easy to see that $f_{Y}$ is universally scalarly measurable also. Hence by Theorem 6 of [8], $f_{Y}$ is Pettis integrable. However, the proof of this theorem yields the fact that $\left\{y f_{Y}: y \in B_{Y}\right\}$ is almost weakly precompact in $L^{\infty}(\mu)$. Hence $f_{Y}$ has the RS property. Since $y f_{Y}=y f$ for every $y \in Y$, we get the desired result.

Although the conclusion to the above proposition is apparently weaker than the function $f$ having the RS-property, it is precisely what is needed to attain the main result of this paper. (See Theorem 5.)

2. Pettis decomposition and the Bourgain property. An interesting property of real-valued measurable functions originally formulated by J. Bourgain [4, 7] will now be employed. Let $(\Omega, \Sigma, \mu)$ be a measure space and $\Psi$ be a family of real-valued functions on $\Omega . \Psi$ is said to have the Bourgain property if for every $E \in \Sigma$ having positive measure and $\varepsilon>0$, there exists a collection $F$ of subsets of $E$ of positive measure such that for every $f \in \Psi$ there exists $B \in F$ such that $\sup f(B)-\inf f(B)<\varepsilon$. The following theorem due to Bourgain [4] (Theorem 11 of $[7])$ demonstrates how strong a property this is.

THEOREM 2. If $(\Omega, \Sigma, \mu)$ is a finite measure space and $\Psi$ is a family of real valued functions on $\Omega$ satisfying the Bourgain property, then

(i) the pointwise closure of $\Psi$ satisfies the Bourgain property,

(ii) each element in the pointwise closure of $\Psi$ is measurable, and

(iii) each element in the pointwise closure of $\Psi$ is the almost everywhere pointwise limit of a sequence from $\Psi$.

Riddle and Saab [7] effectively use the Bourgain property to study the Pettis integral. Let $f: \Omega \rightarrow X^{*}$ be a bounded function. They define the function $f$ to have the Bourgain property if the family $\left\{x f: x \in B_{X}\right\}$ has the Bourgain property. Theorem 13 of [7] states that if $f$ has the Bourgain property then $f$ is indeed Pettis integrable. However, the converse of this theorem is not true. It is shown in Example 14 of [7] that there exists a universally Pettis integrable function without the Bourgain property.

The next results are true for finite separable measure spaces $(\Omega, \Sigma, \mu)$. This means that there exists a sequence $\left(\pi_{n}\right)$ of finite partitions of $\Omega$ of elements of $\Sigma$ such that $\pi_{n+1}$ refines $\pi_{n}$ for every $n \in \mathbf{N}$ and the union of the $\sigma$-algebras generated by these partitions is dense in $\Sigma$.

Let $(\Omega, \Sigma, \mu)$ be a separable measure space, $X$ a Banach space, and $f: \Omega \rightarrow X^{*}$ a bounded and weak ${ }^{*}$ scalarly measurable function. Let $\left(\pi_{n}\right)$ be a sequence of partitions of $\Omega$ as above and let $\Sigma_{n}$ be the $\sigma$-algebra generated by the members of $\pi_{n}$. There is then a natural $X^{*}$-valued sequential martingale $\left(f_{n}, \Sigma_{n}\right)$ associated with $f$, defined by

$$
f_{n}=\sum_{A \in \pi_{n}} \frac{w^{*}-\int_{A} f d \mu}{\mu(A)} \chi_{A} .
$$

We are now ready to state and prove an extension of Theorem 18 of [7] to duals of nonseparable Banach spaces. We first prove the following extension of Le nma 17 of [7]. 
LEMMA 3. Let $(\Omega, \Sigma, \mu)$ be a separable measure space, $X$ be a Banach space, and $f: \Omega \rightarrow X^{*}$ be a bounded and weak $k^{*}$ scalarly measurable function. Let $\left(f_{n}, \Sigma_{n}\right)_{n=1}^{\infty}$ be the sequential martingale associated with $f$ defined by $(*)$. Then there exist functions $g$ and $h$ such that $g$ has the Bourgain property, $h$ is weak $k^{*}$ scalarly null, and $f=g+h$ if and only if the family $\left\{x f_{n}: n \in \mathbf{N}, x \in B_{X}\right\}$ has the Bourgain property.

PROOF. We may assume $f$ takes its range in $B_{X}^{*}$. Suppose that $\left\{x f_{n}: n \in \mathbf{N}\right.$, $\left.x \in B_{X}\right\}$ has the Bourgain property. Since $\left\{f_{n}\right\}_{n=1}^{\infty} \subset\left(B_{X^{*}} \text {, weak }{ }^{*}\right)^{\Omega}$, a compact space, there exists a function $g: \Omega \rightarrow B_{X}^{*}$ such that $g$ is a weak ${ }^{*}$ pointwise cluster point of $\left\{f_{n}\right\}_{n=1}^{\infty}$. By Theorem 2, $g$ has the Bourgain property. However it is easy to see that $x g=x f$ a.e. $\mu$ for every $x \in X$. Hence $h=f-g$ is weak ${ }^{*}$ scalarly null.

Now suppose that $g: \Omega \rightarrow B_{X^{*}}$ has the Bourgain property. If $\left(g_{n}, \Sigma_{n}\right)_{n=1}^{\infty}$ is the sequential martingale associated with $g$ defined by $(*)$, then the proof of Lemma 17 of [7] shows that $\left\{x g_{n}: n \in \mathbf{N}, x \in B_{X}\right\}$ has the Bourgain property. (Note that the separability hypothesis of Lemma 17 of [7] is not used for this part of the proof.) However if $f-g$ is weak* scalarly null, then $g_{n}=f_{n}$ for every $n \in \mathbf{N}$, and hence we get the desired result.

Before we prove the next proposition, we should observe that a function $f$ has the Bourgain property if and only if for every $\varepsilon>0$ there exists $E \in \Sigma$ such that $\mu(\Omega \backslash E)<\varepsilon$ and $f \chi_{E}$ has the Bourgain property [7]. To see this, let $A \in \Sigma$ be such that $\mu(A)=\varepsilon>0$, and apply the Bourgain condition to the nonnull set $A \cap E$, where $E$ satisfies the above.

Proposition 4. Let $(\Omega, \Sigma, \mu)$ be a separable measure space, $X$ a Banach space and $f: \Omega \rightarrow X^{*}$ a bounded weak $k^{*}$ scalarly measurable function. Suppose for every separable subspace $Y$ of $X$ that $\left\{y f: y \in B_{Y}\right\}$ is almost weakly precompact in $L^{\infty}(\mu)$. Then there exist functions $g$ and $h$ such that $g$ has the Bourgain property, $h$ is weak* scalarly null, and $f=g+h$.

PROOF. Consider the evaluation operator $T_{f}: X \rightarrow L^{1}(\mu)$. Let $\left\{x_{n}\right\}_{n=1}^{\infty}$ be a sequence in $B_{X}$ such that $\left\{T_{f}\left(x_{n}\right)\right\}_{n=1}^{\infty}$ is dense in $T_{f}\left(B_{X}\right)$. Let $Z$ be the closed linear span of $\left\{x_{n}\right\}_{n=1}^{\infty}$. Since $Z$ is separable, $\left\{z f: z \in B_{Z}\right\}$ is almost weakly precompact in $L^{\infty}(\mu)$.

Let $\left(f_{n}, \Sigma_{n}\right)_{n=1}^{\infty}$ be the sequential martingale associated with $f$ defined by $(*)$. By Lemma 3 it suffices to show that $\left\{x f_{n}: n \in \mathbf{N}, x \in B_{X}\right\}$ has the Bourgain property. By the observation preceding the statement of this proposition, we may assume that $\left\{z f: z \in B_{Z}\right\}$ is weakly precompact in $L^{\infty}(\mu)$.

Suppose that $\left\{x f_{n}: n \in \mathbf{N}, x \in B_{X}\right\}$ does not have the Bourgain property. We then build a sequence $\left(z_{n}\right)_{n=1}^{\infty}$ in $B_{Z}$, a system $\left(C_{n, m}\right), n \in \mathbf{N}, 1 \leq m \leq 2^{n}$, of sets of positive measure, and constants $\delta<\beta$, such that

(1) $C_{n+1,2 m-1} \subset C_{n, m}$ and $C_{n+1,2 m} \subset C_{n, m}$;

(2) $z_{n+1} f(\omega)<\delta$ if $\omega \in C_{n+1,2 m-1}$;

(3) $z_{n+1} f(\omega)>\beta$ if $\omega \in C_{n+1,2 m}$;

The proof is by induction on $n$ and proceeds as in Theorem 18 of [7] with one modification. At the $n$th stage of the construction, they find an element $x_{n+1}$ in $B_{X}$ such that the sets

$$
A_{n+1,2 m-1}=\left\{\omega \in C_{n, m}: x_{n+1} f(\omega)<\delta\right\}
$$


and

$$
A_{n+1,2 m}=\left\{\omega \in C_{n, m}: x_{n+1} f(\omega)>\beta\right\}
$$

both have positive measure. We need to find an element $z_{n+1}$ in $B_{Z}$ that also possesses this property. We use the fact that $T_{f}\left(B_{Z}\right)$ is $L^{1}(\mu)$ dense in $T_{f}\left(B_{X}\right)$ in order to find a sequence $\left(y_{k}\right)_{k=1}^{\infty}$ in $B_{Z}$ such that $y_{k} f$ converges almost uniformly to $x_{n+1} f$. It is easy to check that if $k$ is large enough, then the sets

$$
C_{n+1,2 m-1}^{k}=\left\{\omega \in C_{n, m}: y_{k} f(\omega)<\delta\right\}
$$

and

$$
C_{n+1,2 m}^{k}=\left\{\omega \in C_{n, m}: y_{k} f(\omega)>\beta\right\}
$$

all have positive measure. Let $z_{n+1}=y_{k}$ for one such $k$.

The rest of the proof follows Riddle and Saab's argument exactly. We then have that $\left\{z_{n} f\right\}_{n=1}^{\infty}$ is isomorphic to the $l^{1}$ basis in $L^{\infty}(\mu)$ norm, contradicting the fact that $\left\{z f: z \in B_{Z}\right\}$ is weakly precompact in $L^{\infty}(\mu)$. Hence $\left\{x f_{n}: n \in \mathbf{N}, x \in B_{X}\right\}$ has the Bourgain property.

3. Universally scalarly measurable functions. We are now able to prove the main theorem of this paper.

THEOREM 5. Let $K$ be a compact Hausdorff space and $X$ be a Banach space. Suppose that $f: K \rightarrow X^{*}$ is bounded and universally scalarly measurable. Then if $\mu$ is a Radon measure on $K$, there exist functions $g$ and $h$ such that $g$ has the Bourgain property for $\mu, h$ is $\mu$-weak* scalarly null, and $f=g+h$. Hence $f$ is $\mu$-Pettis decomposable.

ProOF. Let $\mu$ be a Radon measure on $K$, and let $\Sigma$ be the $\sigma$-algebra of universally measurable subsets of $K$. Let $\Pi$ be the set of finite partitions of $K$ into elements of $\Sigma$ directed by refinement. If $\pi \in \Pi$, let $\Sigma_{\pi}$ be the finite $\sigma$-algebra generated by the elements of $\pi$ and let

$$
f_{\pi}=\sum_{A \in \pi} \frac{w^{*}-\int_{A} f d \mu}{\mu(A)} \chi_{A} .
$$

It is an immediate consequence of Fremlin's Theorem [5] [1] that the evaluation operator $T_{f}: X \rightarrow L^{1}(\mu)$ is compact, and hence $f_{\pi} \rightarrow f$ in Pettis norm by Proposition 2 of [1]. Thus, there exists a sequence $\left\{\pi_{n}\right\}_{n=1}^{\infty}$ in $\Pi$ such that $\pi_{n+1}>\pi_{n}$ for every $n \in \mathbf{N}$ and $f_{\pi_{n}} \rightarrow f$ in Pettis norm. Let $\Sigma_{0}=\sigma-\left(\bigcup_{n=1}^{\infty} \Sigma_{n}\right)$. Since $\left(f_{\pi_{n}}, \Sigma_{\pi_{n}}\right)_{n=1}^{\infty}$ is clearly a bounded martingale, there exists a function $f_{0}: \Omega \rightarrow X^{*}$ such that $x f_{\pi_{n}} \rightarrow x f_{0}$ a.e.- $\mu$ for every $x \in X$. (See Corollary 3.3 of [3].) On the other hand, since $f_{\pi_{n}} \rightarrow f$ in Pettis norm, $x f_{\pi_{n}} \rightarrow x f$ in $L^{1}(\mu)$. Therefore $x f=x f_{0}$ a.e. $\mu$ for every $x \in X$. It is also clear that $f_{0}=$ weak $^{*}-E\left(f \mid \Sigma_{0}\right)$ and thus $f_{0}$ is weak* $^{*}$ scalarly measurable with respect to $\Sigma_{0}$.

Let $Y$ be any separable subspace of $X$. We get that $\left\{y f: y \in B_{Y}\right\}$ is almost weakly precompact in $L^{\infty}(\mu)$ by Proposition 1 . Since conditional expectation operators are $L^{\infty}(\mu)$ contractions, $\left\{y f_{0}: y \in B_{Y}\right\}$ must also be almost weakly precompact in $L^{\infty}(\mu)$. Thus, by Proposition 4 , there exist functions $g$ and $\hat{h}$ such that $g$ has the Bourgain property for $\mu, \hat{h}$ is $\mu$-weak* scalarly null, and $f_{0}=g+\hat{h}$. The desired conclusion is obtained by observing that $h=\hat{h}+f-f_{0}$ is $\mu$-weak ${ }^{*}$ scalarly null. 
We conclude with the following summary:

THEOREM 6. Let $K$ be a compact Hausdorff space, $X$ a Banach space and $f: K \rightarrow X^{*}$ a bounded function. Then the following are equivalent.

(i) $f$ is universally scalarly measurable.

(ii) For every regular Borel measure $\mu$ on $K$, there exists a $\mu$-Pettis integrable function $g$ and a $\mu$-weak $k^{*}$ scalarly null function $h$ such that $h$ is $\mu$-scalarly measurable and $f=g+h$.

(iii) For every regular Borel measure $\mu$ on $K$, there exists functions $g$ and $h$ such that $g$ has the Bourgain Property for $\mu, h$ is $\mu$-weak $k^{*}$ scalarly null and $\mu$-scalarly measurable and $f=g+h$. [7].

In the case $X$ is separable, we get the following strengthening of Corollary 19 of

COROLlaRY 7. Let $K$ be a compact Hausdorff space, $X$ be a separable Banach space, and $f: K \rightarrow X^{*}$ be a bounded function. Then the following are equivalent.

(i) $f$ is universally scalarly measurable.

(ii) $f$ is universally Pettis integrable.

(iii) If $\mu$ is a Radon measure on $K$ then $f$ has the Bourgain property for $\mu$.

ProOF. If $X$ is a separable Banach space then weak* scalarly null functions are zero a.e. (Proposition 1 of [2]). Hence the equivalence of (i), (ii), and (iii) follows from Theorem 5 and Lemma 15 of [7].

The equivalence of (i) and (ii) was given in [8]. The equivalence of (iii) with the other two in the case $K$ is a compact metric space is Corollary 19 of [7].

\section{REFERENCES}

1. E. M. Bator, The Pettis Integral and the equality of the norms of the Dunford integral and the weak* integral, Proc. Amer. Math. Soc. 95 (1985), 265-270.

2. __ A decomposition of bounded scalarly measurable functions taking their ranges in dual Banach spaces, Proc. Amer. Math. Soc. 102 (1988), 850-854.

3. E. M. Bator, P. W. Lewis, and D. Race, Some connections between Pettis integration and operator theory, Rocky Mountain Math. J. 17 (1985), 265-270.

4. J. Bourgain, Martingales in conjugate Banach spaces (Unpublished preprint).

5. D. H. Fremlin, Pointwise compact sets of measurable functions, Manuscripta Math. 15 (1975), 219-242.

6. R. S. Phillips, Integration in a convex linear topological space, Trans. Amer. Math. Soc. 47 (1940), 114-145.

7. L. H. Riddle and E. Saab, On functions that are universally Pettis integrable, Illinois J. Math. 29 (1985), 509-531.

8. L. H. Riddle, E. Saab, and J. J. Uhl Jr., Sets with the weak Radon-Nikodym property in dual Banach spaces, Indiana Univ. Math. J. 32 (1983), 527-540.

9. H. P. Rosenthal, A characterization of Banach spaces containing $l^{1}$, Proc. Nat. Acad. Sci. U.S.A. 71 (1974), 2411-2413.

10. M. Talagrand, Pettis integral and measure theory, Mem. Amer. Math. Soc., No. 307 (1984). 76203

Department of Mathematics, North TeXas State University, Denton, TeXas 